\title{
Isoelektrischer Punkt der Epithelien der Nasen= schleimhaut verschiedener Tiere. ${ }^{1)}$
}

\author{
Von \\ Akira Tamura. \\ (Aus dem Anatomischen Institut der Medizinischen Fakultät zu Okayama ; \\ Vorstand: Prof. K. Yagita und Prof. M. Seki.)
}

Über den isoelektrischen Punkt der Epithelien der Nasen- und Nasennebenhöhlen des Menschen liegt schon eine Arbeit von Seki und Chin (194I) vor. Ich nahm zur Ergänzung analoge Untersuchungen an verschiedenen Tieren vor.

\section{Material und Methode.'}

Das Material wurde verschiedenen Tieren, Kaninchen, Meerschweinchen, Maus, Affe, Katze und Hund entnommen. Die entnommene Schleimhaut liess sich in einem $95 \%$ igen Alkohol fixieren und in Paraffin einbetten. Dann wurden Schnitte von Io $\mu$ Dicke angefertigt. Die Schnitte wurden mit Ponceau (sauer) und Toluidinblau (basisch) bei $\mathrm{pH}$ 2,6-7,0 gefärbt. Die Details der Methode finden sich in der Mitteilung von Seki und Kohashi (1940) und Seki und Chin (1940). Das pHi wurde immer an dem oberflächlichen kernfreien Epithelgebiet gemessen, also in der Regio respiratoria am Zytoplasma der flimmernden Zylinderepithelzellen und in der Regio olfactoria an demjenigen der Stützzellen zusammen mit den oberen Fortsätzen der Riechzellen.

\section{Resultate.}

Die Resultate der Messung lassen sich in folgender Tabelle zusammenstellen.

Aus der Tabelle ersieht man, dass der isoelektrische $\mathrm{pH}$-Wert des weniger dicht gebauten Epithels der Regio respiratoria immer deutlich kleiner ist als

1) Ausgeführt auf Kosten der Ausgaben des Unterrichtsministeriums für wissenschaftliche Forschung. 
pHi der Epithelien der Nasenschleimhaut.

\begin{tabular}{l|c|c}
\hline \multicolumn{1}{c|}{ Tiere } & Regio respiratoria & Regio olfactoria \\
\hline Kaninchen & 4,95 & 5,10 \\
Meerschweinchen & 5,00 & 5,15 \\
Maus & 4,95 & 5,20 \\
Affe & 4,95 & 5,10 \\
Katze & 4,80 & 5,00 \\
Hund & 4,85 & 5,00
\end{tabular}

derjenige des dichter strukturierten Riechepithels. Der Unterschied der Strukturdichte tritt, worauf v. Möllendorf (1924) und Seki (1936) auf-merksam gemacht haben, durch gewisse Färbemethode klar zutage. Z. B., bei der Azanfärbung lassen sich die Epithelzellen der Regio respiratoria stärker blau und schwächer rot färben als der Regio olfactoria. Des weiteren ergibt sich, dass die Nasenschleimhautepithelien von Karnivoren, wie der Katze und des Hundes von einem niedereren $\mathrm{pHi}$-Wert sind gegenüber denjenigen Omni- und Herbivoren, wie des Affen, der Maus, der Meerschweinchens und des Kaninchens.

\section{Zusammenfassung.}

Die Nasenschleimhautepithelien sind überhaupt bei den Fleischfressern von einem niedereren $\mathrm{pHi}$ als bei den Alles- und Pflanzenfressern. Die Epithelien der Regio respiratoria weisen bei verschiedenen Tieren immer ein niedereres $\mathrm{pHi}$ auf als die Riechepithelien.

\section{Literaturverzeichnis.}

Möllendorf, W. u. M. v.: Untersuchungen zur Theorie der Färbung fixierter Präparate. III. Erg. Anat. 25 (1924).

Seki, M.: Zur physikalischen Chemie der histologischen Färbung. XI. Z. Zellforsch. 24 (1936).

Seki, M. u. Y. Kohashi: Bestimmung des isoelektrischen Punktes der Gewebselemente auf färberischem Wege. 1. Okajimás Fol. anat. jap. 19 (1940).

Seki, M. u. K. Chin: Bestimmung des isoelektrischen Punktes der Gewebselemente auf färtberischem Wege. III. Okajimas. Fol. anat. jap. 19 (1940).

Seki, M. u. K. Chin: Bestimmung des isoelektrischen Punktes der Gewebselemente auf färberischem Wege. VII. Z. Zellforsch. 3I (194I). (Im Druck). 